\title{
Transatlantica
}

Revue d'études américaines. American Studies Journal

\section{Isabelle Alfandary. E.E. Cummings. Eric Athenot.} Walt Whitman.

Paris : Belin, 2002. 126 p. Paris : Belin, 2002. 126 p. http://www.editionsbelin.com

\section{Alain Suberchicot}

\section{(2) OpenEdition}

Journals

Édition électronique

URL : http://journals.openedition.org/transatlantica/745

DOI : $10.4000 /$ transatlantica.745

ISSN : $1765-2766$

Éditeur

AFEA

Référence électronique

Alain Suberchicot, «Isabelle Alfandary. E.E. Cummings. Eric Athenot. Walt Whitman. », Transatlantica [En ligne], 1 | 2003, mis en ligne le 05 avril 2006, consulté le 29 avril 2021. URL : http://

journals.openedition.org/transatlantica/745 ; DOI : https://doi.org/10.4000/transatlantica.745

Ce document a été généré automatiquement le 29 avril 2021.

\section{(c) (i) $\odot$}

Transatlantica - Revue d'études américaines est mis à disposition selon les termes de la licence Creative Commons Attribution - Pas d'Utilisation Commerciale - Pas de Modification 4.0 International. 


\section{Isabelle Alfandary. E.E. Cummings. Eric Athenot. Walt Whitman.}

Paris : Belin, 2002.126 p. Paris : Belin, 2002. 126 p. http://www.editionsbelin.com

Alain Suberchicot

1 On doit louer Marc Chénetier pour avoir suscité l'écriture de ces deux volumes consacrés à Cummings et à Whitman, et encouragé ainsi les chercheurs à consacrer leurs travaux à la poésie américaine. Isabelle Alfandary et Eric Athenot s'acquittent avec passion et finesse de la tâche qui leur a été confiée de présenter une synthèse dans un espace relativement restreint. Dans les deux cas, nous sommes devant des ensembles littéraires ambitieux, quoique très différents, et nés d'époques qui ne se ressemblent guère sinon par leurs désastres et l'appel à la générosité humaine et à la responsabilité politique qu'elles inspirent à l'un comme à l'autre poète.

Dans sa lecture de l'œuvre de Cummings, Isabelle Alfandary, on n'en sera guère surpris, s'intéresse à l'écriture, mais elle le fait de telle sorte que c'est la matérialité du signe poétique qui est l'objet de sa réflexion, et plus spécifiquement un jeu dans l'espace, qui inquiète le sens. Est notamment mise en évidence avec brio ce qu'Isabelle Alfandary nomme « la primauté de la sensation », c'est-à-dire l'attrait qu'exerce sur Cummings la venue de la lettre dans une configuration unique et surprenante devenue poème, et qui est l'effet d'un travail d'agencement propre à Cummings. D'où l'idée qui consiste à étudier les poèmes de Cummings en tant qu'événement, question à laquelle est consacré le chapitre 3 de l'ouvrage. C'est à ce point de sa réflexion que le livre d'Isabelle Alfandary atteint un degré particulièrement fascinant lorsqu'elle nous dit, traçant peut-être les contours d'une recherche future, que Cummings cherche à tordre le cou à l'idéalisme des romantiques et que "pour en venir à bout, il faut ré-investir l'idéal romantique, le fissurer de l'intérieur, le faire sonner creux, l'affoler par une grammaire qui a perdu le sens commun, en un mot, l'émouvoir» (55). Une telle perspective critique est particulièrement juste car elle met à jour une volonté de la part de Cummings d'asseoir l'autorité de la poésie en tant que genre, et le meilleur moyen d'y parvenir est effectivement de se tourner vers les romantismes, qui sont un moment 
important de constitution de la poésie. Il reste malgré tout à déterminer si les romantismes eux-mêmes n'avaient pas entrepris avant la modernité littéraire de fissurer l'idéalisme par pure volonté de conscience cognitive. Et Isabelle Alfandary nous restitue la fraîcheur de Cummings, en nous faisant prendre la mesure de l'attention qu'il portait à l'amour, amour des autres, amour de la langue, ce qui tisse un irréductible écheveau de substance relevant effectivement de l'idéalisme. D'où sans doute ce vacillement de la poésie de Cummings, qui n'échappe pas à Isabelle Alfandary, surtout lorsqu'elle en vient à ce moment d'intense acuité intellectuelle qui la porte à décrire comme suit, par une formule qu'on lui envie tant elle ramasse l'analyse, un processus fondamental de l'écriture de Cummings : «Accidenter la langue, l'affecter, c'est faire surgir en elle le sens comme événement ». (72) On lira également avec profit la partie consacré à la voix chez Cummings, qui nous permettra de tirer des enseignements utiles, bien entendu, à la compréhension des ressorts fondamentaux de l'œuvre de Cummings, mais aussi de nombre de poètes du vingtième siècle.

3 Eric Athenot, dans son ouvrage, modifie l'image de Whitman à laquelle nous nous sommes habitués, dans la mesure où l'on connaît trop les moments d'extase panthéiste du grand poète américain, et ses élans d'enthousiasme politique, suscités par Abraham Lincoln, notamment. Tout l'intérêt de la démarche d'Eric Athenot est de faire apparaître la gravité du poète, et les incertitudes qui parcourent son écriture. On doit aussi à Eric Athenot de nous appeler à la lecture des textes fondamentaux, et s'il en est un, c'est bien Varieties of Religious Experience, de William James, qui examine d'un point de vue philosophique les diverses traditions religieuses et comporte des remarques à propos de Walt Whitman. William James a ainsi forgé à propos de Whitman, nous l'apprenons, la notion d'émotion ontologique (" a passionate and mystic ontological emotion, » Library of America, 83). Eric Athenot fait ensuite travailler cette notion dans son étude, et aboutit lui-même à la notion d'utopie onirique, qui ne manque pas d'intérêt, puisque, effectivement, il y a une part d'échappée vers l'irréel chez Whitman, assurément pour combattre l'angoisse, et maitriser ce qu'elle peut avoir de déstabilisant quand on recherche comme le fait Whitman avec la poésie une religion de la bonne santé de l'esprit («a religion of healthy-mindedness », c'est la formule de William James pour décrire les vitalistes parmi les chrétiens, ceux qui ne sont pas alourdis par la faute). De là provient la nécessité qu'il y avait à tempérer l'optimisme de James, qui s'abat sur Whitman, et a tendance à nous enfermer dans le type de lecture qu'Eric Athenot s'emploie à combattre, à juste titre. Car la notion d'utopie onirique semble confirmer l'idée de James d'une émotion ontologique, et la combat adroitement sans le dire, au nom d'une compréhension du lyrisme poétique, et contre la philosophie qui a la fâcheuse tendance à voir dans la poésie un exercice de cruciverbiste, en général soit plaintif soit enjoué, sans portée réelle. Un chapitre du livre d'Eric Athenot prend une direction semblable, qui consiste à montrer que la poésie est une revendication de sens, le chapitre consacré à la pensée politique de Whitman. Les arguments sont maîtrisés, et le propos fondamental d'Eric Athenot nuancé, et là aussi ramassé en une saisissante formule, lorsqu'il évoque «l'alliage troublant de pessimisme désenchanté et d'optimisme millénariste » (73).

4 Ces deux ouvrages démontrent la rigueur et l'enthousiasme de la recherche française en matière de poésie américaine, et une capacité de positionnement théorique qui fait notre force. 
INDEX

Thèmes : Recensions

\section{AUTEUR}

\section{ALAIN SUBERCHICOT}

Université Jean-Moulin - Lyon 3 